Revista de Psicología y Educación / Journal of Psychology and Education, 2022, 17(1), 1-20 (www.rpye.es) Doi: https://doi.org/10.23923/rpye2022.01.213

ISSN: $1699-9517$

\title{
Construcción de una escala sobre igualdad de género percibida en la escuela
}

\author{
Ignacio Moya-Díaz y Ángel De-Juanas
}

Escuela Internacional de Doctorado. Programa de Educación. Universidad Nacional de Educación a Distancia, Madrid, España

Resumen: La estimación con pruebas estandarizadas sobre la creencia del profesorado acerca de la situación actual de la igualdad de género en las aulas resulta de interés para establecer predicciones sobre la práctica real de comportamientos sexistas en los centros educativos. El objetivo de este trabajo fue validar una escala para conocer, desde la valoración subjetiva del profesorado, cuáles son los comportamientos presentes en la escuela que actúan como transmisores de contenidos sexistas en el currículum oculto. Para la construcción de este instrumento se realizó una revisión inicial por parte de 11 expertos y un estudio piloto en el que participaron 221 docentes de centros educativos de la Comunidad de Madrid. Se determinó la fiabilidad y validez de la escala. Los resultados evidenciaron que la consistencia interna del instrumento arrojó un valor de $\alpha$ de Cronbach superior a ,84. El análisis factorial mostró cinco factores que explicaron el $54,5 \%$ de la varianza. Las propiedades psicométricas de la escala fueron adecuadas para medir la percepción del profesorado sobre comportamientos transmisores de contenidos sexistas en la escuela. Con ello, se abre una línea de investigación futura para detectar, mediante este instrumento, creencias y corregir comportamientos sexistas en los centros educativos.

Palabras clave: Igualdad de género, Currículum oculto, Escala de creencias, Profesorado, Estereotipos.

\section{Construction of an equality gender scale perceived in education}

Abstract: The estimation with standardized tests on the teachers' belief about the current situation of gender equality in the classrooms is of interest to establish predictions about the real practice of sexist behaviors in schools. The objective of this work was to validate a scale to know, from the subjective assessment of the teachers, the behaviors present in the school that act as transmitters of sexist content in the hidden curriculum. For the construction of this instrument, an initial review was carried out by 11 experts. Then, a pilot study, in which 221 teachers from educational centers in the Community of Madrid participated, were conducted. The reliability and validity of the scale was examined. The results showed that the internal consistency of the instrument generated a Cronbach's $\alpha$ value higher than .84 . The factor analysis showed five factors that explained $54.5 \%$ of the variance. The psychometric properties of the scale were adequate to measure teachers' perception of behaviors that transmit sexist content at school. Thus, a future line of research is opened to detect beliefs by means of this instrument, and to correct sexist behaviors in educational centers.

Key words: Gender equality, Hidden curriculum, Education, Teacher's perception, Stereotypes.

La Agenda 2030 ha planteado un ambicioso plan de objetivos para lograr durante la presente década entre los que destaca especialmente conseguir la igualdad

Recibido: 09/06/2021 - Aceptado: 12/11/2021 - Avance online:13/12/2021

*Correspondencia: Ignacio Moya Díaz-Agero

Escuela Internacional de Doctorado. Programa de Educación. Universidad Nacional de Educación a Distancia, Madrid, España Dirección: C/Juan del Rosal 14. 28040, Madrid, España.

E-mail: imoya34@alumno.uned.es

Moya-Díaz, I. y De-Juanas, A. (2022). Construcción de una escala sobre igualdad de género percibida en la escuela. Revista de Psicología y Educación, 17(1), 1-20, hitps://doi. org/10.23923/rpye2022.01.213 entre los géneros y empoderar a todas las mujeres y las niñas. En esta hacendosa tarea, la familia, la escuela y las administraciones educativas adquieren un papel nuclear. Una labor que debe construirse desde varios ámbitos: político-educativo; trasmisión de los valores socioculturales en la escuela; y, la formación del profesorado (UNESCO, 2020). 
En las últimas décadas, las políticas educativas sobre la igualdad de género han estado presentes en la legislación española. Sin embargo, en los referentes normativos de carácter educativo se relega la igualdad de género a un asunto interdisciplinary transversal que no se asume de forma particular como contenido directo de ningún área o materia (González, 2018). Esta falta de concreción deja en manos del profesorado la decisión de incorporarla a sus programaciones (Martínez García, 2007). En esta línea debe amplificarse la estrategia del gendermainstreaming, que sitúa a todas las políticas bajo la perspectiva de género para evitar el androcentrismo. Esta última estrategia pretende superar algunos obstáculos fuertemente arraigados a la cultura educativa, siendo uno de ellos la persistencia de normativas que permiten la segregación por sexos en los centros educativos. La existencia de estos modelos educativos se opone a la idea de convertir la escuela en un reflejo de la sociedad, un microcosmos, en el que el estudiante interactúa con una sociedad pluralista. En suma, tampoco existe una justificación clara de sus beneficios, ya que como algunos autores señalan: el rendimiento escolar aumenta cuando el alumnado se expone a una mayor interacción educativa y cultural con otros agentes sociales" (Rubia, 2013, p.6).

Otro aspecto relevante, sobre el que se estructura la igualdad de género en la escuela, son los valores socioculturales que se relacionan con ella y que permanecen activos en la sociedad. En ellos se insertan los estereotipos, las creencias y los prejuicios sexistas. Esta tendencia sobre la percepción de la igualdad de género en la enseñanza "evidencia que los docentes prestan más atención a los niños varones en el aula, y que desconocen sus propias actitudes sexistas, teniendo poca conciencia de cuándo y cómo transmiten estereotipos de género a sus alumnos" (Mencía-Ripley, 2020, p. 4).

Asimismo, las creencias socioculturales también derivan en actitudes que influyen sobre parámetros aún más complejos y que se encuentran presentes en la escuela -véase el ejemplo de la brecha digital de género o el techo de cristal -.(Vico y Rebollo, 2019).

En cualquiera de los casos, se debe amplificar el estudio y seguimiento de estos aspectos, ya que los estudios realizados hasta la fecha muestran que "(...) a través del material didáctico y las interacciones sociales en el contexto escolar se van aprendiendo, reforzando y manteniendo estereotipos de género" (Basto y Soberanis, 2020, p.5).

Por su parte, la formación del profesorado también se ha visto señalada como un área influyente en la igualdad de género. Al respecto, Miralles-Cardona et al. (2020) aplicaron la Escala de Evaluación Sensible a la Formación en Igualdad de Género (ESFIG) a 601 docentes de formación universitaria y hallaron una escasa integración de la percepción de género en la docencia universitaria. En la misma línea, otros estudios apuntan a la escasa importancia que se le otorga a la igualdad de género en el currículum universitario (Garrigues, 2010; Larrondo y Rivero, 2019; Valdivieso et al., 2016; Verge et al., 2018). Por su parte, Pastor-Gosálbez et al. (2020) consideran que la formación universitaria ha integrado desarrollos normativos que fomentan la igualdad de género pero, que en la práctica se hacen invisibles. Del mismo modo, otros trabajos internacionales apuntan a la falta de esfuerzos por abordar la igualdad de género en la formación del profesorado desde las universidades (Atchison, 2013; Lahelma y Tainio, 2019; Zippel et al., 2016).

\section{EL CURRÍCULUM OCULTO Y EL GÉNERO}

El currículum educativo se corresponde con un plan de estudios, una declaración de intenciones que establece las bases del conocimiento que han de adquirir los estudiantes para convertirse en ciudadanos de una sociedad plural, democrática y justa. Sin embargo, en las interacciones y en la experiencia de enseñanza-aprendizaje que ocurre en la escuela, se pone de manifiesto la importancia de las lecciones, valores, 
perspectivas que no están escritas, pero que se comunican de manera implícita por parte del profesorado (Santomé, 1991). Tal y como advirtió González (1996), el currículum oculto influye en la educación y directamente en la construcción del ideario de género. Desde esta premisa surgió el término currículum oculto de género (en adelante, COG) (Dorr y Sierra, 1998). Se trata de un constructo en el que se interpreta la igualdad de género en la escuela como un parámetro que opera fuertemente influenciado y conectado al currículum oculto; entendido a su vez como "el conjunto de normas, actitudes, expectativas, creencias y prácticas que se instalan de forma inconsciente en las estructuras y el funcionamiento de las instituciones y en el establecimiento y desarrollo de la cultura hegemónica de las mismas" (Santos, 2002, p.3).

La estructura interna sobre la que se edifica el COG invita a señalar tres ejes principales sobre los que se asienta este constructo: androcentrismo, determinismo biológico y polarización del género (Dorr y Sierra, 1998).

El primero de ellos, androcentrismo, responde teóricamente a la visión del mundo diseñado por y para los hombres e impregna los contenidos científicos que se imparten en las aulas, así como el análisis de su transmisión desde los libros de texto (Bello, 2002). Este eje se extiende a toda la cultura, las doctrinas, las instituciones, los valores sociales, los valores religiosos e incluso las deidades. En todos ellos se refleja la creencia de la superioridad masculina (Strenski, 2021).

El segundo eje, determinismo biológico, comprende que el comportamiento humano depende de aspectos genéticos difícilmente modificables $y$, en consecuencia:

"el hecho de que el género se base en la diferencia sexual sigue dando por hecho que el sexo es natural. Esta relación entre sexo y género aparece como dos categorías que dependen una de la otra. La segunda es analizada como la construcción social de la primera (el género) y la primera (el sexo) se asume como un hecho pre-existente" (Curiel, 2014 , p. 9).

Por ende, se asumen patrones de conducta propios de cada uno de los géneros, como por ejemplo la realización de gestos obscenos por un exceso de testosterona en el sexo masculino.

El tercero y último, tiene que ver con la polarización del género y se centra en la teorización de aquellos motivos por los que los hombres y mujeres son diferentes. Este eje pretende organizar todo el sistema cultural y educativo sobre la base de este hecho.

\section{INSTRUMENTOS SOBRE IGUALDAD DE GÉNERO EN EDUCACIÓN}

El estudio de la igualdad de género en educación ha sido un tema ampliamente abordado por numerosas investigaciones (Alvariñas-Villaverde, Gallargo-López y Gallardo-Vázquez, 2019; Prendes-Espinosa et al., 2020). Sin embargo, resulta notoria la escasez de instrumentos específicos diseñados sobre esta temática. Este hecho se agrava aún más, cuando se considera también el constructo 'currículum oculto'. Sin embargo, existen algunas herramientas que han tratado de acercarse a esta temática de estudio. Así, surgieron los primeros cuestionarios y escalas que sirvieron de impulso para diagnosticar el estado de la igualdad de género en la sociedad $y$, posteriormente, en la escuela (Rigat-Pflaum, 2008). Entre estos destacaron la Escala de Identidad de Género Masculino de Blanchard y Freund (1983) y el trabajo de Alumbaugh (1987). Este último contrastó la herramienta de Blanchard y Freund con la Escala 5-MF (MaleFemale) de la primera versión del Inventario Multifásico de la Personalidad de Minnesota (MMPI) que fue diseñada por Hathaway y McKinley (1981). Una década después, se desarrolló la Escala sobre las creencias de los Roles de Género (GRBS) impulsada por Kerr y Holden (1996) y que estudiaba las creencias sobre el desarrollo de los roles de género en graduados universitarios. Estos primeros estudios sirvieron para corroborar 
cuánto había evolucionado la percepción de la construcción de género distanciándose, cada vez más, de las tradicionales conexiones que asociaban los problemas de identidad, homosexualidad o la realidad transgénero a las psicopatologías (Rigat-Pflaum, 2008). En cualquiera de los casos, por primera vez, estas primeras escalas analizaron la identidad de género y definieron en su conjunto los roles de género, pero en su desarrollo no contemplaron explícitamente la igualdad de género como un factor mesurable $y$, mucho menos, ligado al ámbito educativo en las primeras edades.

Desde entonces, emergieron estudios que dieron lugar a nuevos instrumentos relacionados con el ámbito educativo y que abordaban la cuestión de género desde diversos puntos de vista. Así, se vincula explícitamente al alumnado, al profesorado de diferentes niveles educativos e incluso a determinadas áreas del currículo como ocurre con Educación Física, pero en ningún caso se diseñó una herramienta que tuviera un planteamiento holístico, integrador y válido para todas las etapas educativas. No obstante, uno de los instrumentos más destacados fue la Escala de Actitudes del Profesorado hacia la Coeducación que presenta tres subescalas - a saber: sociocultural, relacional e individualy se dirige de manera específica a docentes de primaria y secundaria de la asignatura de Educación Física (Piedra et al., 2014). Con idéntica estructura factorial y orientada para alumnos de $5^{\circ}$ y $6^{\circ}$ de Educación Primaria y $1^{\circ}$ y $2^{\circ}$ de Educación Secundaria, Pérez et al. (2010) construyeron la Escala de Actitudes hacia la lgualdad de Género dirigida al Alumnado. Este instrumento fue replicado en otros trabajos con notable éxito (Polo, 2020). Igualmente, son reseñables la Escala de Juegos Deportivos y Emociones (GES) para alumnos de secundaria (Gözütok et al., 2017) y la Escala de Detención del Sexismo en Adolescentes (DSA), diseñada por Recio et al. (2007) y que fue aplicada con éxito en estudios posteriores (Bernuy y Grijalva, 2017; Burgos, 2018; Cueva, 2017; Fernandes et al., 2020).
Desde el ámbito universitario surgieron otros instrumentos como la Escala de aprendizaje en las redes sociales virtuales que aborda la igualdad de género desde el constructo brecha digital de género (Vico y Rebollo, 2019) o el cuestionario Actitudes hacía la lgualdad de Género (ACTIG), en donde se organiza la percepción de género de los estudiantes universitarios desde cuatro dimensiones - global, relacional, sociocrítica y personal- (Castillo et al., 2020).

En consecuencia, tras una revisión exhaustiva de los instrumentos existentes relacionados con la temática, consideramos que la diversidad de estudios e instrumentos no ha permitido abordar con suficiencia y especificidad la estructura interna del constructo de igualdad de género cuando se vincula al currículum oculto en el ámbito educativo. Tampoco se reconoce un instrumento de medida validado entre la literatura hispanohablante que posibilite el diagnóstico y evaluación del currículum oculto mediante los aspectos más relevantes asociados a la igualdad de género en relación con el profesorado. Menos aún, existe un instrumento que englobe la percepción del constructo desde el enfoque de todas las etapas educativas. Por consiguiente, se requiere un nuevo enfoque que no repita los fundamentalismos que han sustentado el uso de la ideología de género desde la perspectiva de los instrumentos que han intentando analizarla (Amaya, 2017).

Con todo, la ausencia de un instrumento específico que aborde la igualdad de género desde la percepción del currículum oculto sugiere la necesidad de diseñar una escala sobre Igualdad de Género Oculta en Educación (en adelante, IGOE). El diseño de este instrumento pretende recoger aquellas dimensiones del currículum oculto que actúan como transmisoras de estereotipos y actitudes sexistas en los escenarios de la escuela y el aula desde la percepción del profesorado. Este trabajo tiene por objetivo presentar un estudio psicométrico que recoge evidencias sobre la construcción de un instrumento válido y confiable sobre la igualdad de género percibida en la escuela. 


\section{MÉTODO}

\section{PARTICIPANTES}

Para determinar la validez de contenido del instrumento participaron 11 jueces y expertos que revisaron los 82 ítems que constituían la propuesta inicial de la escala. Entre los jueces hubo seis profesores universitarios que impartían docencia en Educación, Psicología, Sociología y Estadística; así como cinco docentes de etapas educativas iniciales que presentaron una amplia experiencia en cargos de gestión docente y en políticas sociales de instituciones públicas educativas. Todos ellos, expertos en la temática de la escala y/o profesionales en ejercicio.

Por otro lado, para analizar la confiabilidad y la validez del instrumento se utilizaron dos submuestras, una para la realización de un estudio piloto y otra para su validación final. En la primera submuestra, relacionada con el estudio piloto, participaron 21 docentes de Educación Infantil, Primaria y Secundaria, de los cuáles 13 fueron mujeres $(61,9 \%)$ y 8 varones $(38,1 \%)$ con edades comprendidas entre los 24 y 65 años $(M=38,6$; DT $=12,03)$. Asimismo, 13 pertenecían a la escuela pública $(N=61,9 \%), 6$ a la concertada $(N=28,6 \%)$ y 2 a la privada $(9,5 \%)$ de la Comunidad de Madrid.

Para la validación factorial de la escala, se contó con una submuestra de 200 docentes, $84,2 \%$ mujeres y $15,4 \%$ de hombres (Perdido: ,04\%) con edades comprendidas entre los 21 y los 63 años $(M=38$; DT $=10,8)$. Este profesorado impartía docencia en diferentes etapas educativas, a saber: Infantil $(\mathrm{N}=21,7 \%)$, Primaria $(\mathrm{N}=58,8 \%)$, Secundaria $(\mathrm{N}=11,7 \%)$ y Bachillerato ( $N=7,9 \%)$. Del mismo modo, los docentes pertenecían a la escuela pública $(\mathrm{N}=$ $89,2 \%)$, a la escuela concertada $(N=4,6 \%)$ y a la escuela privada ( $N=6,3 \%)$. La experiencia docente de los participantes se distribuía de manera homogénea entre los que tenían menos de 5 años de experiencia( $N=28,3 \%)$, los que tenían entre 5 y 15 años ( $N=27,5 \%)$ y los que presentaba más de 15 años de experiencia $(\mathrm{N}=34,2 \%)$. Para determinar la muestra necesaria en este estudio, se optó por escoger un mínimo de cinco participantes por ítem del cuestionario siguiendo el criterio de Nunnally y Bernstein (1994).

\section{INSTRUMENTO}

La construcción de la escala IGOE ha formado parte de una investigación más extensa que tiene como objetivo determinar, desde la valoración subjetiva del profesorado, cuáles son los comportamientos presentes en la escuela que actúan como transmisores de contenidos sexistas en el currículum oculto. Para la elaboración de este instrumento, se realizó una revisión del estado de la cuestión en torno al objeto de estudio. La escala debía contrastar las percepciones del profesorado sobre la realidad educativa y establecer puentes de conexión con el currículum oculto. Para ello, se decidió que iría destinada a un amplio espectro de docentes pertenecientes a las etapas comprendidas desde Educación Infantil a Bachillerato. El instrumento se diseñó para cuestionar a los docentes por su percepción sobre diferentes hechos $y / 0$ situaciones asociadas a la igualdad de género que tienen lugar en el ámbito educativo. En consecuencia, la escala diseñada fue de tipo Likert y presentó cinco posibles alternativas de respuestas que fueron desde totalmente en desacuerdo (1) hasta completamente de acuerdo (5).

Los ítems que se tomaron como punto de partida para la elaboración de la escala se diseñaron a partir de la revisión de otros cuestionarios e instrumentos en los que la igualdad de género se agrupó en torno a diferentes factores: la gobernanza e influencia de las administraciones en las políticas de igualdad, el empoderamiento de la mujer (autonomía personal, recursos económicos, valores socioculturales), la organización social responsable y la violencia contra las mujeres (Alfama et al., 2014).

Igualmente, se tomó como referencia la Escala de Actitudes del Profesorado hacia la Coeducación (Rebollo et al., 2011). De 
la misma forma, se realizó una revisión sistemática de la literatura científica sobre el constructo de igualdad de género (PrendesEspinosa et al.,2020) y sobre los factores que inciden en su desarrollo para las diferentes áreas escolares (Alvariñas-Villaverde, PazosGonzález, 2018). Se consideró el trabajo de Mellizo-Soto (2014) en el que se analizó exclusivamente la situación de la igualdad de género dentro del territorio español.

En consecuencia, las variables diseñadas de forma preliminar para la construcción del instrumento se agruparon en cinco grandes ejes temáticos: la igualdad de género y la normativa; la igualdad de género y la influencia socio cultural; la igualdad de género y las metodologías docentes; la igualdad de género en las relaciones interpersonales entre docentes y discentes; y, finalmente, la igualdad de género en la actividad física y el deporte. Estas cinco categorías fueron asumidas inicialmente como potenciales factores. Tomando las recomendaciones de otros estudios (Vallejo et al., 2003), se definieron y conceptualizaron estos cinco ejes temáticos como dimensiones iníciales para, posteriormente, servir de pauta en la elaboración final de la escala. En cualquier caso, estas dimensiones no se perfilaron con un interés confirmatorio sino como una propuesta descriptiva y exploratoria que permitiese configurar, con posterioridad, una escala robusta desde el punto de vista de la validez de constructo.

\section{PROCEDIMIENTO}

Se desarrollaron tres estudios exploratorios, el primero con un diseño no experimental descriptivo en el que tras la elaboración de las variables objeto de medición se realizó un primer cribado de ítems a partir de la aplicación del instrumento al juicio de una muestra de 11 expertos que analizaron la validez de contenido. El segundo estudio constituye la aplicación del instrumento a una muestra piloto que permitió completar el análisis de la confiabilidad y validez de contenido del instrumento. El tercer y último estudio consistió en la aplicación de la escala a una muestra de 200 participantes y permitió obtener evidencias sobre su estructura factorial.

\section{LA CONSTRUCCIÓN DEL CONJUNTO INICIAL DE ÍTEMS}

Inicialmente, se creó un pool de ítems que comprendió un total de 114 elementos que recogían prejuicios, creencias, estereotipos, actitudes, o connotaciones sexistas vinculados al COG. Una vez generado este banco de ítems, se realizaron varias revisiones por parte de los investigadores donde, tras discriminar las variables por su representatividad con los constructos a medir, se cribaron los ítems hasta reducir el pool de ítems a un total de 82.

\section{ESTUDIO DE LA VALIDEZ DE CONTENIDO}

Se facilitó a 11 jueces una tabla de registro donde debían valorar la claridad, relevancia y pertinencia de cada uno de los 82 ítems finalistas. Una vez recogidos los datos, se utilizó el modelo de Lawshe (1975) para determinar la razón de validez de contenido (RVC) de cada uno de ellos. Como resultado de estas acciones se redujo la escala a 46 reactivos.

\section{ESTUDIO PILOTO}

Los 46 elementos seleccionados se organizaron de forma aleatoria y se configuró la escala para ser aplicada en un estudio piloto. La prueba se aplicó mediante papel y lápiz, previo consentimiento informado de los participantes. Se estuvo presente durante su aplicación, se midió el tiempo de realización y se anotaron las incidencias. Posteriormente, se modificó la redacción de varios ítems a partir de las valoraciones que hicieron los participantes. Se constató que el tiempo medio de realización de la escala fue de 6 minutos y producto de estas actuaciones la escala se redujo a 40 ítems.

\section{ESTUDIO DEL ANÁLISIS FACTORIAL EXPLORATORIO}

Finalmente, se realizó un segundo estudio con el propósito de recoger datos 
que permitieran llevar a cabo los análisis estadísticos pertinentes y proceder con el diseño factorial de la escala. Para ello, se aplicó de nuevo el instrumento a una muestra de mayor tamaño $(N=200)$ en formato de papel y lápiz y también por administración online mediante un formulario de Google. En todo momento, se respetó la confidencialidad siguiendo el protocolo establecido por la Declaración de Helsinki (64 ${ }^{a}$ AMM, Brasil, octubre de 2013) y los participantes fueron informados del propósito de la investigación y dieron su conformidad para formar parte de ella.

\section{ANÁLISIS DE DATOS}

Para realizar el análisis de razón de validez de contenido (RVC) de Lawshe se utilizó el programa Microsoft Excel del paquete Office 365. Para el análisis de fiabilidad se recurrió al estadístico a de Cronbach, también se realizó un análisis factorial exploratorio, todo ello mediante el programa informático IBM SPSS 27.0 para Windows.

\section{RESULTADOS}

\section{VALIDEZ DE CONTENIDO}

Tras la recogida de datos de los 11 jueces, se estimaron como aceptables todos los valores situados por encima de ,637, valor referenciado según el modelo de Lawshe (op. Cit.) para cada ítem. Posteriormente, se midió la RVC ajustada a cada parámetro de cada variable (claridad, relevancia y pertinencia). Como resultado de este estudio, se revisaron y modificaron los ítems que daban lugar a controversia por presentar un lenguaje poco discriminante, y se redujo el cuestionario a 46 ítems. En ese momento se obtuvo un índice de validez de contenido total de la escala de ,888.

\section{ESTUDIO PILOTO}

Se analizaron los estadísticos descriptivos y la fiabilidad de la escala. Inicialmente, se obtuvo un coeficiente $\alpha$ de Cronbach de
,825. Posteriormente, se revisaron aquellos ítems que mejoraban el coeficiente $\alpha$ de Cronbach con su retirada. Se decidió eliminar 6 reactivos y modificar la redacción de otros 3. Estas actuaciones redujeron la escala a 40 elementos. La fiabilidad de la escala arrojó un nuevo coeficiente $\alpha$ de Cronbach de ,897 que se tomó con prudencia a la espera de su comportamiento ante una encuestación mayor.

\section{ANÁLISIS FACTORIAL EXPLORATORIO}

Previamente al análisis factorial exploratorio (en adelante, AFE), se midió de nuevo la consistencia interna de la escala en su aplicación a 200 participantes. Se obtuvo una $\alpha$ de Cronbach de ,802. Con el propósito de mejorar la fiabilidad, un análisis ítem a ítem permitió reducir la escala a 32 elementos. Esta decisión permitió aumentar la consistencia de la escala hasta un $\alpha$ de Cronbach de ,844. Este nivel de consistencia interna fue aceptable y permitió desarrollar el AFE con la intención de obtener un modelo teórico de partida que explicase cómo podrían categorizarse en un nivel conceptual las variables del estudio. Su aplicación facilitó analizar cómo se producía la condensación de los ítems en factores dentro de nuestra escala. Para ello, se identificaron el número de factores que explicaron la máxima proporción de varianza común a todas las variables en tres fases: una inicial donde se aplicó el método de componentes principales, una posterior donde se empleó un método de rotación oblicua y otra final donde se produjo un análisis y reflexión de los datos emergidos y su categorización conceptual. Se consideró esencial observar el comportamiento de la escala frente al método de componentes principales, la intención fue valorar cómo respondía la varianza total de la escala ante las varianzas específica de cada ítem y los posibles errores de aplicación. Se realizaron algunos ajustes en la propia escala que pasaron por eliminar 13 reactivos (de los 32 anteriores) que arrojaron bajas puntuaciones ( $p$ $<0,6$.) en la matriz anti-imagen. Paralelamente, se comprobó de nuevo la consistencia interna de escala y se obtuvo un $\alpha$ de Cronbach de ,841. Se clarifica la evolución de este estadístico en la Tabla 1. 
Tabla 7

Evolución de la Consistencia Interna de la Escala durante su Construcción

\begin{tabular}{|c|c|c|}
\hline Medición de la fiabilidad & Participantes & $\alpha$ de Cronbach \\
\hline En prueba piloto con la escala de 46 reactivos & 21 &, 825 \\
\hline En prueba piloto con la escala reducida a 40 reactivos & 21 &, 802 \\
\hline En segunda muestra con la escala reducida a 40 reactivos & 200 &, 844 \\
\hline En segunda muestra con la escala reducida a 32 reactivos & 200 &, 841 \\
\hline En la escala final con 19 reactivos tras realizar el AFE & & 200 \\
\hline
\end{tabular}

Posteriormente, se ejecutó el AFE y se aplicó el método de rotación oblicua Promax (Tabla 3) por considerar la posible relación de los factores. La medida de Kaiser-MeyerOlkin ofreció un valor de ,832. La prueba de esfericidad de Barlett posibilitó realizar el análisis factorial y descartar la hipótesis nula de que la matriz fuese una matriz identidad $(p<, 001)$. Por su parte, el determinante de la matriz de correlaciones desprendió como valor de ,002, situándose en valores cercanos a cero lo que indicó altas intercorrelaciones entre variables. Igualmente, se comprobó que la comunalidad de los ítems superase ,30 (Hair et al., 2004). Finalmente, se analizó la varianza total explicada y se agruparon los elementos sobre 5 factores. Estos factores saturaron con auto valores superiores a 1 . La agrupación en torno a 5 factores explicó el 54,58\% (véase Tabla 2).

Seguidamente, se verificó la agrupación de factores en la matriz rotada por el método de rotación oblicua promax.

Tabla 2

Varianza Total explicada

\begin{tabular}{|c|c|c|c|c|c|c|c|}
\hline \multirow{2}{*}{ Componente } & \multicolumn{3}{|c|}{ Autovalores iniciales } & \multicolumn{3}{|c|}{$\begin{array}{l}\text { Sumas de cargas al cuadrado de la } \\
\text { extracción }\end{array}$} & \multirow{2}{*}{$\begin{array}{c}\text { SCCR* } \\
\text { Total }\end{array}$} \\
\hline & Total & $\begin{array}{c}\% \text { de } \\
\text { varianza }\end{array}$ & $\begin{array}{c}\% \\
\text { acumulado }\end{array}$ & Total & $\begin{array}{c}\% \text { de } \\
\text { varianza }\end{array}$ & $\begin{array}{c}\% \\
\text { acumulado }\end{array}$ & \\
\hline 1 & 4,97 & 26,16 & 26,16 & 4,97 & 26,16 & 26,16 & 3,04 \\
\hline 2 & 1,72 & 9,10 & 35,26 & 1,72 & 9,10 & 35,26 & 3,41 \\
\hline 3 & 1,43 & 7,57 & 42,83 & 1,43 & 7,57 & 42,83 & 3,12 \\
\hline 4 & 1,20 & 6,33 & 49,17 & 1,20 & 6,33 & 49,17 & 2,49 \\
\hline 5 & 1,02 & 5,41 & 54,58 & 1,02 & 5,41 & 54,58 & 2,27 \\
\hline
\end{tabular}




\begin{tabular}{|l|}
\hline Tabla 2 (Continuación) \\
Varianza Total explicada \\
\hline
\end{tabular}

\begin{tabular}{|c|c|c|c|c|c|c|c|}
\hline \multirow{2}{*}{ Componente } & \multicolumn{3}{|c|}{ Autovalores iniciales } & \multicolumn{3}{|c|}{$\begin{array}{l}\text { Sumas de cargas al cuadrado de la } \\
\text { extracción }\end{array}$} & \multirow{2}{*}{$\begin{array}{c}\text { SCCR* } \\
\text { Total }\end{array}$} \\
\hline & Total & $\begin{array}{c}\% \text { de } \\
\text { varianza }\end{array}$ & $\begin{array}{c}\% \\
\text { acumulado }\end{array}$ & Total & $\begin{array}{c}\% \text { de } \\
\text { varianza }\end{array}$ & $\begin{array}{c}\% \\
\text { acumulado }\end{array}$ & \\
\hline 6 &, 94 & 4,97 & 59,56 & & & & \\
\hline 7 & ,90 & 4,77 & 64,33 & & & & \\
\hline 8 & 84 & 4,45 & 68,78 & & & & \\
\hline 9 &, 75 & 3,98 & 72,77 & & & & \\
\hline 10 & ,73 & 3,84 & 76,61 & & & & \\
\hline 11 & ,69 & 3,65 & 80,26 & & & & \\
\hline 12 &, 65 & 3,44 & 83,70 & & & & \\
\hline 13 &, 58 & 3,06 & 86,76 & & & & \\
\hline 14 &, 53 & 2,83 & 89,59 & & & & \\
\hline 15 &, 47 & 2,47 & 92,07 & & & & \\
\hline 16 &, 42 & 2,21 & 94,29 & & & & \\
\hline 17 & 41 & 2,18 & 96,47 & & & & \\
\hline 18 &, 35 & 1,88 & 98,36 & & & & \\
\hline 19 &, 31 & 1,63 & 100,00 & & & & \\
\hline
\end{tabular}

Tabla 3

Matriz con Rotación Oblicua por el método Promax

Factores

\begin{tabular}{|c|c|c|c|c|c|}
\hline & 1 & 2 & 3 & 4 & 5 \\
\hline 1.38 & 0,819 & 0,274 & 0,327 & 0,287 & 0,132 \\
\hline 1.39 & 0,795 & 0,367 & 0,135 & 0,141 & 0,181 \\
\hline 1.40 & 0,730 & 0,469 & 0,423 & 0,173 & 0,181 \\
\hline 1.35 & 0,602 & 0,166 & 0,385 & 0,051 & 0 \\
\hline
\end{tabular}


Tabla 3 (Continuación)

Matriz con Rotación Oblicua por el método Promax

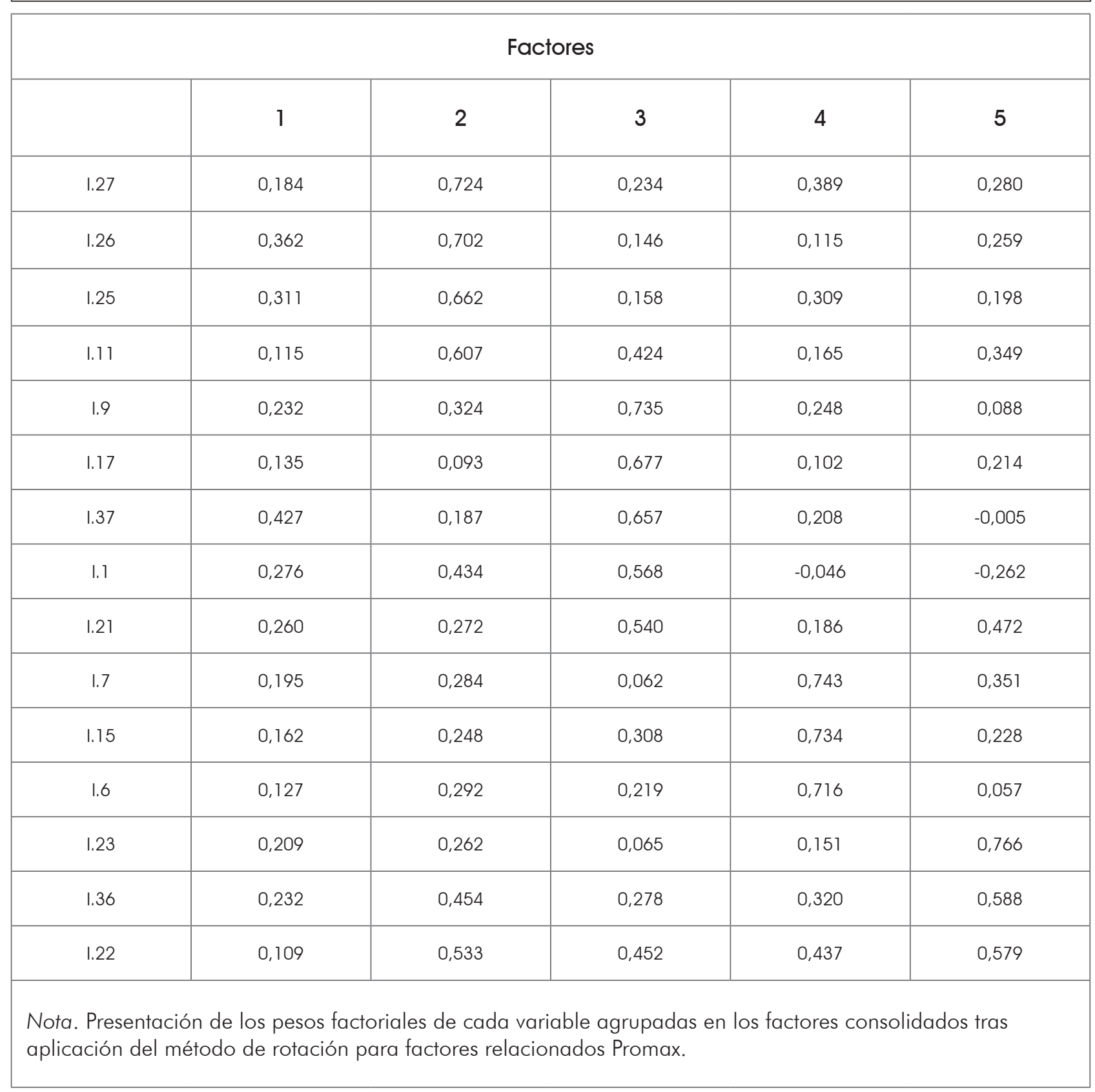

El análisis de datos concluyó con la configuración final de la escala compuesta por un total de 19 elementos con una consistencia interna global de ,841. Del análisis factorial se obtuvo una agrupación de los ítems en 5 factores que fueron asumidas como dimensiones conceptuales del estudio. Se procuró llevar a cabo un correcto ajuste teórico de las evidencias emanadas del AFE. Así mismo se estudió la consistencia interna de cada subescala (Tabla 4).

\section{DIMENSIONES DE LA ESCALA}

1. Predominancia Masculina (PM). Constituida por 4 ítems $(35,38,39,40)$ en la que se agrupan secuencias de transmisión del currículum oculto a través de las creencias, hechos o situaciones que se asocian en exclusiva al género masculino y en donde se reflejan las tendencias de género en la escuela. En algunos casos estas conexiones se derivan de las relaciones docente-discente y discente 
- discente. El coeficiente $\alpha$ de Cronbach de la PM fue de, 748 .

\section{Determinismo Biológico (DB).} Dimensión conformada por 4 ítems $(11,25$, $26,27)$ identifica lo que el profesorado espera sobre el comportamiento de cada género por su influencia genética. El coeficiente $\alpha$ de Cronbach de la DB fue de ,709.

3. Liderazgo e lgualdad de Género (LIG). Compuesta por 5 ítems $(1,9,17,21,37)$. Se valora la percepción del liderazgo en la educación desde una perspectiva social, relacional e institucional. El coeficiente $\alpha$ de Cronbach de la LIG fue de ,701.
4. Situación de Género en la Escuela (SGE). Esta dimensión está constituida por tres ítems $(6,7,15)$. Se recogen diferentes percepciones y estereotipos que el profesorado manifiesta sobre los roles de género en la escuela y en la sociedad. Todos ellos actúan como límites de la igualdad de género. El coeficiente $\alpha$ de las SGE fue Cronbach ,698.

5. Predominancia Femenina (PF), constituida por 3 ítems $(22,23,36)$. Señala las secuencias de transmisión del currículum oculto a través de las creencias, hechos o situaciones que se asocian a las fortalezas y debilidades del género femenino y en donde se reflejan las tendencias de género en la escuela. El coeficiente $a$ de Cronbach de la PF fue de , 636 .

Tabla 4

Dimensión de la Escala IGOE

\begin{tabular}{|c|c|c|c|c|}
\hline Factor & $\mathrm{N}^{\mathrm{O}}$ & Ítem & Peso & Alpha* \\
\hline \multirow{4}{*}{$\begin{array}{c}1 \\
\text { PM }\end{array}$} & 35 & $\begin{array}{l}\text { Los enfoques competitivos en el aula consolidan la } \\
\text { identidad de género. ** }\end{array}$ & ,602 & \multirow{4}{*}{$\alpha=, 748$} \\
\hline & 38 & $\begin{array}{c}\text { En mis clases los chicos suelen querer recibir primero el } \\
\text { material. }\end{array}$ & 819 & \\
\hline & 39 & Los chicos siempre están más motivados en mis clases. & ,795 & \\
\hline & 40 & $\begin{array}{c}\text { Generar un ambiente en clase en el que se fomente la } \\
\text { participación de forma competitiva beneficia más a los } \\
\text { chicos. }\end{array}$ & ,730 & \\
\hline \multirow{4}{*}{$\begin{array}{c}2 \\
\mathrm{DB}\end{array}$} & 11 & $\begin{array}{l}\text { Prefiero tener una tutoría con una madre que con un } \\
\text { padre. }\end{array}$ & 607 & \multirow{4}{*}{$\alpha=, 709$} \\
\hline & 25 & Los chicos generan más problemas en los patios. & 622 & \\
\hline & 26 & $\begin{array}{l}\text { Los problemas y dificultades ocasionados por los chicos } \\
\text { son más fáciles de resolver. }\end{array}$ & ,702 & \\
\hline & 27 & $\begin{array}{c}\text { Es más probable que un gesto obsceno sea realizado } \\
\text { por un chico. }\end{array}$ &, 724 & \\
\hline
\end{tabular}




\begin{tabular}{|c|c|c|c|c|}
\hline \multicolumn{5}{|c|}{$\begin{array}{l}\text { Tabla } 4 \text { (Continuación) } \\
\text { Dimensión de la Escala IGOE }\end{array}$} \\
\hline Factor & No & lítem & Peso & Alpha* \\
\hline \multirow{5}{*}{$\begin{array}{l}3 \\
\text { LIG }\end{array}$} & 1 & $\begin{array}{l}\text { La educación que mis estudiantes reciben en sus } \\
\text { hogares está basada en un modelo dirigido a favorecer } \\
\text { a los chicos. }\end{array}$ &, 568 & \multirow{5}{*}{$\alpha=, 701$} \\
\hline & 9 & $\begin{array}{l}\text { El puesto de dirección en los centros educativos suele } \\
\text { estar ocupado por un hombre. }\end{array}$ & ,735 & \\
\hline & 17 & El lenguaje utilizado en las aulas no es inclusivo. &, 677 & \\
\hline & 21 & $\begin{array}{l}\text { El liderazgo en mis clases suele ser asumido por algún } \\
\text { chico. }\end{array}$ &, 540 & \\
\hline & 37 & El coordinador TIC en los centros suele ser un hombre. & 657 & \\
\hline \multirow{3}{*}{$\begin{array}{c}4 \\
S G E\end{array}$} & 6 & A mis tutorías suelen acudir más madres que padres. &, 716 & \multirow{3}{*}{$\alpha=, 698$} \\
\hline & 7 & $\begin{array}{c}\text { Tras la vuelta del patio los chicos suelen volver más } \\
\text { desaliñados y sucios que las chicas. }\end{array}$ & ,743 & \\
\hline & 15 & $\begin{array}{l}\text { Los chicos utilizan con mayor frecuencia las pistas } \\
\text { polideportivas de los centros escolares durante el tiempo } \\
\text { de recreo. }\end{array}$ & ,734 & \\
\hline \multirow{3}{*}{$\begin{array}{c}5 \\
\mathrm{SPF}\end{array}$} & 22 & $\begin{array}{l}\text { Las chicas recogen mejor y más cuidadosamente el } \\
\text { material del aula que los chicos. }\end{array}$ & .579 & \multirow{3}{*}{$\alpha=, 6363$} \\
\hline & 23 & $\begin{array}{c}\text { Cuando necesito que un estudiante me ayude a mover } \\
\text { un material pesado prefiero elegir a un chico. }\end{array}$ & .766 & \\
\hline & 36 & $\begin{array}{l}\text { Los chicos son más chapuceros que las chicas al hacer } \\
\text { las tareas. }\end{array}$ &, 588 & \\
\hline \multicolumn{5}{|c|}{$\begin{array}{l}\text { Nota. Consistencia interna de cada dimensión. Tabla de elaboración propia. } \\
\text { *El término Alpha hace referencia al estadístico } \alpha \text { de Cronbach } \\
\text { **Se ha reducido el contenido del elemento } 35 \text {, excluyendo el paréntesis aclaratorio que incorpora la versión } \\
\text { final del instrumento }\end{array}$} \\
\hline
\end{tabular}

\section{DISCUSIÓN}

La construcción de la Escala IGOE se ha desarrollado con el objetivo de señalar los elementos presentes en la escuela que actúan como transmisores de contenido sexistas a través del Currículum Oculto de Género. Se configura como una herramienta fiable y concreta, que además responde a las demandas educativas y a la igualdad de género en todas las etapas educativas; al tiempo que permite dar cabida a la presencia del currículum oculto en la transmisión de los estereotipos y las actitudes sexistas presentes en el sistema educativo.

En primera instancia debe aclarase que el estudio no pretendía verificar un modelo teórico ya existente con la aplicación de un Análisis Factorial Confirmatorio (AFC), sino abrir una nueva línea de factores que explicasen como se comportaba el constructo igualdad de género cuando conectaba con el currículo oculto. Algunas certezas se evidenciaron 
por las siguientes razones: se trató de una escala de nueva creación; se desconocía la estructura factorial subyacente; no se disponía de otras escalas con las que comparar la estructura factorial de la Escala IGOE puesto que ninguna contemplaba la presencia del constructo currículum oculto, ni tampoco otras muestras sobre las que se hubiese aplicado la propia escala. En consecuencia, no se pudieron realizar las pruebas de bondad de ajuste que son requeridas en los AFC.

El diseño de IGOE se llevó a cabo en un constante ajuste de los parámetros que la han constituido, conformándose inicialmente por 114 reactivos, frente a los 19 ítems finales. Este cribado evidenció la importancia de definir con claridad los pilares que edifican la igualdad de género en la escuela como punto de partida para incidir en su análisis (Castillo et al., 2020).

Tras su diseño se obtuvieron evidencias de la consecución de los objetivos de la investigación. La fiabilidad del instrumento final mostró en su conjunto una consistencia interna notoria ( $\alpha$ de Cronbach de, 841 ) y una validez de contenido meritoria (RVC ,888). Estos datos han arrojado corolarios particularmente parejos a los de otros instrumentos diseñados hasta la fecha en temática de igualdad de género (Del-Castillo, 2009; Cervelló et al., 2004; Mercado, 2005). Del análisis de la fiabilidad de las subescalas han emergido datos prudentes, pero aceptables ( $\alpha$ de PM ,748; $\alpha$ de DB ,709; $\alpha$ de LIG ,701; $\alpha$ de SGE ,698; $\alpha$ de PF ,636). Debe considerarse con igual importancia las consistencias internas de cada dimensión y la consistencia interna total del instrumento.

Por su parte, el AFE permitió reagrupar los 19 reactivos finales de la Escala IGOE en cinco factores que han posibilitado definir un modelo teórico estructural donde se fijan los descriptores que pueden actuar como transmisores del COG. Las 19 variables se estructuraron en torno a cinco factores que explicaron el 54,5\% de la varianza total explicada. Esta disposición detalla, concreta y ajusta otras propuestas de agrupación realizadas con anterioridad en otros estudios sobre igualdad de género en donde, a pesar de no incorporar el constructo currículum oculto, todas las variables fueron organizadas en tres categorías: relacional, sociocultural y personal (García-Pérez et al., 2010; Piedra et al., 2014). En consecuencia, la agrupación de los reactivos reflejó una estructura factorial distinta a la de estos modelos y, por tanto, los resultados se deben tomar con prudencia y como una presentación preliminar de los posibles constructos que explican la influencia del currículum oculto sobre la igualdad de género.

En cualquiera de los casos, el principal hito de este estudio corrobora la existencia de cinco dimensiones que constituyen el modelo teórico que subyace a la construcción y a la transmisión de la igualdad de género mediante el currículum oculto. Estas dimensiones se habían encontrado en la literatura científica asociándose a la igualdad de género pero nunca de forma conjunta. Así se destacan; el determinismo biológico (Martín, 2007), la predominancia masculina (Mata et al., 2013), la situación del género en la escuela (Moreno, 2000; Murillo y Martínez, 2018), liderazgo e igualdad de género (Díaz et al., 2017; Lomas, 2007; Martínez, 2020) y la predominancia de la mujer (Fernández-Antón, 2021). De todas ellas, las tres primeras dimensiones de la Escala IGOE han presentado garantías de fiabilidad y validez como para constituirse como ejes nucleares del modelo teórico a desarrollar. Sin embargo, las dos últimas, requieren una revisión más exhaustiva con posteriores iteraciones.

Paralelamente, se ha de señalar que no se han encontrado escalas o instrumentos que midiesen los constructos igualdad de género y currículum oculto de forman aunada, por lo que con la Escala IGOE se abre un nuevo campo de estudio que permite esclarecer vinculaciones entre estos elementos. Su aplicación en diferentes contextos educativos deberá servir de pauta para reconocer y describir las percepciones del profesorado en la trasmisión del COG. 
Este estudio no está exento de limitaciones. Se debe reconocer que todo análisis factorial exploratorio puede tener una interpretación más descriptiva que explicativa, siendo un procedimiento para generar y probar teorías más que para confirmarlas. En consecuencia, sus resultados son siempre más conservadores. Como principal condicionante ha de indicarse el grupo incidental que fue utilizado como muestra. A su vez, y a pesar de obtenerse una distribución heterogénea de los participantes, nos hemos encontrado con una pequeña representación de profesorado de la escuela no pública y del profesorado que sólo imparte docencia en la etapa de Bachillerato. Por consiguiente, en estudios futuros, se debe considerar la necesidad de aplicar la Escala IGOE en otras muestras que dispongan de una mayor representatividad de la escuela concertada y privada. Estas acciones serán relevantes para ejecutar un posterior análisis factorial confirmatorio que permita consolidar la estructura factorial del modelo teórico emergido de este estudio.

\section{- Conflicto de intereses}

Los autores declaran no tener ningún conflicto de interés.

\section{REFERENCIAS}

Alfama, E., Cruells, M. y De La Fuente, M. (2014). Medir la igualdad de género. Debates y reflexiones a partir de una propuesta de sistema de indicadores clave. Athenea Digital. Revista de pensamiento e investigación social, 14(4), 209-235. https://doi.org/10.5565/rev/ athenea. 1460

Alumbaugh, R. V. (1987). Contrast of the Gender-identity Scale WithBem's Sexrole Measures and the MF Scale of the MMPI. Perceptual and Motor Skills, 64(1), 136. https://doi.org/10.2466/ pms.1987.64.1.136

Alvariñas-Villaverde, M. y Pazos-González, M. (2018). Estereotipos de género en Educación Física, una revisión centrada en el alumnado. Revista electrónica de investigación educativa, 20(4), 154-163. https://doi.org/10.24320/ redie.2018.20.4.1840

Amaya, J. F. S. (2017). La tormenta perfecta: Ideología de género y articulación de públicos. Sexualidad, Salud y Sociedad, (27), 149-171. https://doi.org/10.1590/1984-6487. sess.2017.27.09.a

Area-Moreira, M., Hernández-Rivero, V., \& Sosa-Alonso, J. J. (2016). Modelos de integración didáctica de las TIC en el aula. Comunicar: Revista Científica de Comunicación y Educación, 24(47), 7987. https://doi.org/10.3916/C47-2016$\underline{08}$

Asociación Médica Mundial (AMM) (21 de marzo de 2017). Declaración de Helsinki de la AMM. Principios éticos para las investigaciones médicas en seres humanos. https://bit.ly/2X6ISOC.

Atchison, A. (2013). The practical process of gender mainstreaming in the political science curriculum. Politics and Gender 9(2), 228-235. https://doi.org/10.1017/ S1743923X13000081

Azorín-Abellán, C.M. (2017). Actitudes hacia la igualdad de género en una muestra de estudiantes de Murcia. Revista Complutense de Educación, 28(1), 45-60. https://doi.org/10.5209/rev RCED.2017.v28.n1.48715

Basto, P. B. P. y Soberanis, N. G. H. (2020). El currículum oculto de estereotipos de género en adolescentes de secundaria. Etic@net: Revista científica electrónica de Educación y Comunicación en la Sociedad del Conocimiento, 20(2), 211 241. https://doi.org/10.30827/eticanet. v20i2.15787

Bello, A. S (2002). El androcentrismo científico: el obstáculo para la igualdad de género en la escuela actual. Educar, (29), 091 - 162. https://doi.org/10.5565/ rev/educar.331

Bernuy, B. J. y Grijalva, H. M. (2017). Sexismo y homofobia en los adolescentes de una institución educativa pública. Propósitos y Representaciones, 5(2), 245-275. http:// dx.doi.org/10.20511/pyr2017.v5n2.162

Blanchard, R. y Freund, K. (1983) Midiendo la identidad de género masculina en mujeres. Revista de consultoría y psicología clínica, 51, 205 - 214 . https:// doi.org/10.33898/rdp.v10i40.791

Burgos, A. R. (2018). Sexismo y violencia en 
las relaciones de noviazgo en adolescentes de una institución educativa pública de la ciudad de Trujillo. (Trabajo de Grado). Universidad Privada del Norte. Trujillo.

Castillo, H. P., Henríquez, M. A. y Tubay, F. (2020). Validación de un Instrumento para evaluar Actitudes hacia la lgualdad de Género en Estudiantes Universitarios. Revista San Gregorio, (38), 100-118. http://dx.doi.org/10.36097/rsan. v1i38.1256

Cervelló, E., Jiménez, R., Del Villar, F., Ramos, L. y Santos-Rosa, F. J. (2004). Goal orientations, motivational climate, equality, and discipline of Spanish physical education students. Perceptual and Motor Skills, 99, 271-283. https:// doi.org/10.2466/pms.99.1.271-283

Cueva, D. E. (2017). Propiedades psicométricas de la escala de detección de sexismo en adolescentes de secundaria del distrito de Florencia de Mora. (Tesis predoctoral), Universidad César Vallejo, Perú. Recuperado de: https://dialnet.unirioja.es/servlet/ articulo? codigo $=2333741$

Curiel, O. (2014). Género, raza, sexualidad: debates contemporáneos. Universidad del Rosario. https://repositorio.unal.edu.co/ handle/unal/75237

Del-Castillo, O. (2009). Evaluación de los factores psicosociales y didácticos relacionados con la equidad de género en Educación Física. Tesis Doctoral. Universidad de Sevilla, Sevilla, España. http://hdl.handle.net/1 1441/15702

Díaz, K. D. C. M., González, R. I. S., Gutiérrez, M. D. L. Á. G. y Durán, E. R. V. (2017). Rompiendo con los Estereotipos: Una experiencia educativa con enfoque de género en una escuela básica. REXE. Revista de Estudios y Experiencias en Educación, 16(32), 165-174. https://www. redalyc.org/pdf/2431/243153684011. pdf

Dorr, A.L. y Sierra, G. (1998). El currículum oculto de género. Educar. Revista de educación, 7, El curriculum oculto de género

Fernandes, M. I. D., Moreira, I. M. P., Silva, A. M., Sá, M. C., Veríssimo, C. M. F. u Leitão, M. N. C. (2020). Escala de
Detección de Sexismo en Adolescentes: tradução e validação para o contexto português. Revista de Enfermagem Referência, 5(1), e19075. https://doi. org/10.12707/RIV19075

Fernández-Antón, E. (2021). La mujer casada y el papel de la educación para su crecimiento personal: análisis de las publicaciones de la escuela moderna (1901-1917). História da Educação, $25 . \quad$ https://doi.org/10.1590/22363459/100443

García-Pérez, R., Rebollo, M. Á., Vega, L., Barragán-Sánchez, R., Buzón, O. y Piedra, J. (201 1). El patriarcado no estransparente: competencias del profesorado para reconocer desigualdad. Cultura y Educación, 23(3), 385-397. https://doi. org/10.1174/113564011797330298

Gallardo-López. J.A. \& GallardoVázquez, P. (2019). Educar en igualdad: prevención de la violencia de género en la adolescencia. Hekademos: Revista educativa digital, (26), 31 -39. https://bit. ly/2z6CBbv

Garrigues, A. (2010). Proyecto integración de la perspectiva de género y las enseñanzas en materia de igualdad de mujeres y hombres y no discriminación en los planes de estudio de grado de la Universitat Jaume I. Resolución de la Dirección General del Instituto de la Mujer de 13 de julio de 2009 (BOE, 22/07/2009). https://repositori.upf.edu/bitstream/ handle/10230/35002/Ventura inte. pdf? sequence $=1$ \&isAllowed $=y$

González Pérez, T. (2018). Políticas Educativas Igualitarias en España. La Igualdad de Género en los Estudios de Magisterio. Archivos Analíticos de Políticas Educativas, 26(2), 1-21. https:// doi.org/10.14507/epaa.26.2764

González, J. I. B. (1996). Cultura profesional y currículum (oculto) en educación física. Reflexiones sobre las (im) posibilidades del cambio. Revista de educación, 52(1), 1349. http://hdl.handle.net/11162/71633 Gözütok, F. D., Toraman, Ç. \& Acar-Erdol, T. (2017). Development of gender equality scale. Elementary Education Online, 16(3), 1036-1048. https://doi. org/10.17051/ilkonline.2017.330240 
Hathaway, S. R. \& McKinley, J. C. (1981). Inventario multifásico de la personalidad. MMPI. Manual. México: Manual Moderno.

Hegarty, P. \& BSarter, E. (2021). The Social Psychology of Sex and Gender. Oxford Research Encyclopedia of Psychology. https://doi.org/10.3389/ fpsyg.2018.01320

Hill, D. B. \& Willoughby, B. L. B. (2005). The Development and Validation of the Genderism and Transphobia Scale. Sex Roles: A Journal of Research, 53(7-8), 531-544. http://dx.doi.org/10.1007/ s $11199-005-7140-x$

Kerr, P. S. \& Holden, R. R. (1996). Development of the gender role beliefs scale (GRBS). Journal of Social Behavior and Personality, 17(5), 3. http://doi. org/10.5923/i.iipbs.20120205.05

Lahelma, E. \&Tainio, L. (2019). The long mission towards gender equality in teacher education: Reflections from a national project in Finland. Nordic Studies in Education, 39(1), 69-84. https://doi. org/10.18261/issn.1891-2019-01-06

Larrondo, A. \& Rivero, D. (2019). A case study on the incorporation of gender-awareness into the university journalism curriculum in Spain. Gender and Education, 31(1), 1-14. https:// doi.org/10.1080/09540253.2016 .1270420

Lawshe, C. H. (1975). A quantitative approach to content validity. Personnel psychology, 28(4), 563-575. https:// doi.org/10.1111/i.1744-6570.1975. tb01393.x

Lomas, C. (2007). ¿̇La escuela es un infierno? Violencia escolar y construcción cultural de la masculinidad. Revista de educación, 342(1), 83-101. https://dialnet.unirioja. es/servlet/articulo? codigo $=2252481$

Lucariello, E. y Caldera, M. I. F. (2011). Prevención de la Violencia de Género en los Adolescentes. International Journal of Developmental and Educational Psychology. Revista de Psicología, 5(1), 113-121. https://dialnet.unirioja.es/ servlet/articulo? codigo $=5876592$

Martín, O. (2007). ¿̇Sexualidad en la escuela?. Buenos Aires, Argentina:
Editorial SB.

Martínez García, J. S. (2007). Clase social, género y desigualdad de oportunidades educativas. Revista de Educación, 342, 287-306. https://dialnet.unirioja.es/ servlet/articulo? codigo $=2254199$

Martínez Lirola, M. (2020). Hacia una educación inclusiva: formación del profesorado de primaria enmarcada en los ODS que potencian la igualdad de género. Revista Iberoamericana de Educación. 2020, 82(2), 27-45. https:// doi:10.35362/rie8223596

Mata, I. M., Ros, C. R., Torróntegui, A. B. y Royuela, C. M. (2013). Estereotipos de sexo y raza en las imágenes de los libros de texto de educación física en primaria. Retos. Nuevas tendencias en educación física, deporte y recreación, (23), 14 18. https://doi.org/10.47197/retos. v0i23.34560

Mellizo-Soto, M. F. (2014). La evolución de la desigualdad de oportunidades educativas: una revisión sistemática de los análisis del caso español. Revista Española de Investigaciones Sociológicas (REIS), 147(1), 107-1 17. https://dialnet.unirioja. es/servlet/articulo? codigo $=4773607$

Mencía-Ripley, A. (2020). Aceptación de la violencia de género en docentes de escuelas públicas. RECIE. Revista Caribeña De Investigación Educativa, 4(1), 81-91. https://doi.org/10.32541/ recie.2020.v4i1.pp81-91

Mercado, H. (2005). Actitud de los Profesores de Educación Física frente a una modalidad mixta de Enseñanza. Tesis Doctoral. Temuco: Universidad de la Frontera.

Merle, Pierre (2012), La ségrégationscolaire. La Découverte.

Miralles-Cardona, C.; Cardona-Moltó, M.C. y Chiner, E. (2020). La perspectiva de género en la formación inicial docente: estudio descriptivo de las percepciones del alumnado. Educación XX1, 23(2), 231-257, https://doi.org/10.5944/ educXX1.23899

Moreno, E. (2000). La transmisión de modelos sexistas en la escuela. El harén pedagógico. Perspectiva de género en la organización escolar, 11 - 
31. https://dialnet.unirioja.es/servlet/ libro? codigo $=1033$

Muñoz, A. P., Campos, J. M. L., Toro, E. O. y de Los Fayos, E. J. G. (2012). Programa para el desarrollo de actitudes de igualdad de género en clases de educación física en escolares. Educación XX1, 15(2), 271-291. https://doi. $\mathrm{org} / 10.5944 /$ educxx 1 15.2.142

Murillo, F. J., \& Martínez Garrido, C. (2018). Magnitud de la segregación escolar por nivel socioeconómico en España y sus Comunidades Autónomas y comparación con los países de la Unión Europea. Revista de la Asociación de Sociología de la Educación (RASE) 17 (1), 37-58. https://doi.org/10.7203/ RASE. 11.1.10129

Kline, R. B. (1999). Nunnally, J. C. \& Bernstein, I. H. (1994). Psychometric theory. Journal of Psychoeducational Assessment, 17, 275-280. https://doi.or $\mathrm{g} / 10.1177 / 014662169501900308$

Pastor-Gosálbez, I., Acosta-Sarmiento, A., Torres-Coronas, T. y Calvo-Merino, M. (2020). Los planes de igualdad en las universidades españolas: situación actual y retos de futuro. Educación $X X 1, \quad 23(1), \quad 147-172 . \quad$ https://doi. org/10.5944/educXX1.23873

Paita, Y. G. (2019). Propiedades psicométricas de la Escala de Detección de Sexismo en Adolescentes (DSA) del séptimo ciclo de instituciones educativas públicas. Tesis predoctoral. Universidad Cesar Vallejo, Perú, Lima. https://dialnet.unirioja.es/servlet/ articulo? codigo $=2333741$

Pérez, R. G., Catalán, M. A. R., García, O. B., González-Piñal, R., Sánchez, R. B. y Pinto, E. R. (2010). Actitudes del alumnado hacia la igualdad de género. Revista de investigación educativa, 28(1), 217-232. http://hdl.handle.net/11441/43556

Piedra, J., García-Pérez, R., FernándezGarcía y E. Rebollo, M. A. (2014). Brecha de género en educación física: actitudes del profesorado hacia la igualdad. Revista Internacional de Medicina y Ciencias de la Actividad Física y del Deporte/ International Journal of Medicine and Science of PhysicalActivity and Sport,
14(53), 1-21. https://revistas.uam.es/ rimcafd/article/view/3914

Pinel-Martínez, C., Pérez-Fuentes, M. C. y Carrión-Martínez, J. J. (2019). Relación entre género, resiliencia y autoconcepto académico y social en la adolescencia. Relationship between gender, resilience, academic and social self-concept in adolescence. Journal of Psychology and Education, 14(2), 112-123, doi: https:// doi.org/10.23923/rpye2019.02.176

Polo, A. Y. (2020). Propiedades psicométricas de la escala de actitudes hacia la igualdad de género en estudiantes de primaria (Tesis predoctoral). Universidad Cesar Vallejo, Chimbote.

Prendes-Espinosa, M. P., García-Tudela, P. A. y Solano-Fernández, I. M. (2020). Igualdad de género y TIC en contextos educativos formales: Una revisión sistemática. Comunicar: Revista Científica de Comunicación y Educación, 28(63), 9-20. https://doi.org/10.3916/ C63-2020-01

Recio, P., Cuadrado, I. y Ramos, E. (2007). Propiedades psicométricas de la Escala de Detección de Sexismo en Adolescentes (DSA). Psicothema, 19(3), 522-528. https://doi.org/10.1037/t00700-000

Rigat-Pflaum, M. (2008). Gender mainstreaming: un enfoque para la igualdad de género. Nueva sociedad, 218, 40. https://dialnet.unirioja.es/ servlet/articulo? codigo $=3988541$

Rubia, F. A. (2013). La segregación escolar en nuestro sistema educativo. In Forum Aragón: Revista Digital de FEAE-Aragón sobre organización y gestión educativa (No. 10, pp. 47-52). Forum Europe de Administraciones de Educación-Aragón. https://dialnet.unirioja.es/servlet/ articulo? codigo $=4688639$

Santomé, J. T. (1991). El curriculum oculto. Ediciones Morata.

Santos, M. Á. G (2002). Currículum oculto y construcción del género en la escuela. Kikiriki. Cooperación Educativa, (4243), 14-27. https://doi.org/10.30827/ eticanet.v20i2.15787

Strenski, I. (2021). Sex and Gender. The Wiley Blackwell Companion to the Study of Religion, 429-439. https://doi. 
$\mathrm{org} / 10.1002 / 9781119092797 . \operatorname{ch} 30$

UNESCO (2015b). A guide for gender equality in teacher education: Policy and practices. https://bit.ly/38nsNGG

UNESCO (2020). Una nueva generación: 25 años de esfuerzos en favor de la igualdad de género en la educación. https://unesdoc.unesco.org/ark:/48223/ pf0000375470

Valdivieso, S., Ayuste, A., RodríguezMenéndez, M.C. y Vila-Merino, E. (2016). Educación y género en la formación docente en un enfoque de equidad y democracia. Democracia y educación en la formación docente (pp. 117- 140). Universidad de Vic-Universidad Central de Cataluña. https://dialnet.unirioja.es/ servlet/articulo? codigo $=5783797$

Vallejo, P. M., Sanz, B. U. y Blanco, A. B. (2003). Construcción de escalas de actitudes tipo Likert. La Muralla.

Verge, T., Ferrer-Fons, M. y González, M.
J. (2018). Resistance to mainstreaming gender into the higher education curriculum. European Journal of Women'sStudies, 25(1), 86-101. https:// doi.org/10.1177/1350506816688237

Vico, A. y Rebollo, M. Á. (2019). El aprendizaje de las mujeres sobre internet y redes sociales: validación y resultados generales de una escala. Educación $X X 1, \quad 22(1), \quad 375-400$. https://doi. $\mathrm{org} / 10.5944 /$ educxx 1.21469

Zippel, K., Ferree, M.M. \& Zimmermann, K. (2016). Gender equality in German Universities: Vernacularising the battle for the best brains. Gender and Education, 28(7), 867-885. https://doi.org/10.108 $\underline{0} 0$ 


\begin{tabular}{|c|c|c|c|c|}
\hline $\begin{array}{c}\text { Totalmente en } \\
\text { Desacuerdo } \\
\text { TD }\end{array}$ & $\begin{array}{c}\text { Algo en } \\
\text { Desacuerdo } \\
\text { AD }\end{array}$ & $\begin{array}{c}\text { Indiferente. Ni en } \\
\text { acuerdo ni en } \\
\text { desacuerdo: I }\end{array}$ & $\begin{array}{c}\text { Algo de Acuerdo } \\
\text { A }\end{array}$ & $\begin{array}{c}\text { Totalmente de } \\
\text { Acuerdo } \\
\text { TA }\end{array}$ \\
\hline
\end{tabular}

\begin{tabular}{|c|c|c|c|c|c|c|}
\hline & Ítem & TD & $A D$ & 1 & A & TA \\
\hline 1 & $\begin{array}{c}\text { La educación que mis estudiantes reciben en sus hogares está basada en un modelo } \\
\text { dirigido a favorecer a los niños. }\end{array}$ & 1 & 2 & 3 & 4 & 5 \\
\hline 2 & $\begin{array}{c}\text { Los medios de comunicación influyen en los valores y las actitudes que imitan los } \\
\text { estudiantes. }\end{array}$ & 1 & 2 & 3 & 4 & 5 \\
\hline 3 & $\begin{array}{l}\text { La docencia en las primeras etapas (infantil, primaria y secundaria) suele ser realizada } \\
\text { mayoritariamente por mujeres y en los niveles más altos por hombres (universidad y FP). }\end{array}$ & 1 & 2 & 3 & 4 & 5 \\
\hline 4 & Las chicas y los chicos son igual de capaces. & 1 & 2 & 3 & 4 & 5 \\
\hline 5 & $\begin{array}{l}\text { Existen limitaciones ocultas que impiden a las mujeres alcanzar puestos de liderazgo } \\
\text { en la sociedad. }\end{array}$ & 1 & 2 & 3 & 4 & 5 \\
\hline 6 & A mis tutorías suelen acudir más madres que padres. & 1 & 2 & 3 & 4 & 5 \\
\hline 7 & $\begin{array}{l}\text { Tras la vuelta del patio los chicos suelen volver más desaliñados y sucios que las } \\
\text { chicas. }\end{array}$ & 1 & 2 & 3 & 4 & 5 \\
\hline 8 & $\begin{array}{l}\text { Por lo general los estudiantes más brillantes de un centro educativo o Universidad } \\
\text { suelen ser hombres. }\end{array}$ & 1 & 2 & 3 & 4 & 5 \\
\hline 9 & El puesto de dirección en los centros educativos suele estar ocupado por un hombre. & 1 & 2 & 3 & 4 & 5 \\
\hline 10 & $\begin{array}{l}\text { Las labores de limpieza y comedor en mi centro escolar son realizadas } \\
\text { mayoritariamente por mujeres. }\end{array}$ & 1 & 2 & 3 & 4 & 5 \\
\hline 11 & Prefiero tener una tutoría con una madre que con un padre. & 1 & 2 & 3 & 4 & 5 \\
\hline 12 & $\begin{array}{c}\text { La segregación por sexos en la escuela empeora el rendimiento académico de los } \\
\text { estudiantes. }\end{array}$ & 1 & 2 & 3 & 4 & 5 \\
\hline 13 & $\begin{array}{l}\text { La normativa educativa no debería permitir la existencia de centros que segregasen } \\
\text { por sexos. }\end{array}$ & 1 & 2 & 3 & 4 & 5 \\
\hline 14 & Las leyes educativas atienden con suficiencia la igualdad de género. & 1 & 2 & 3 & 4 & 5 \\
\hline 15 & $\begin{array}{l}\text { Los chicos utilizan con mayor frecuencia las pistas polideportivas de los centros } \\
\text { escolares durante el tiempo de recreo. }\end{array}$ & 1 & 2 & 3 & 4 & 5 \\
\hline 16 & $\begin{array}{c}\text { Cuando necesito ayuda para realizar una tarea prefiero elegir a quien mejor la pueda } \\
\text { desempeñar sin importancia del género. }\end{array}$ & 1 & 2 & 3 & 4 & 5 \\
\hline 17 & El lenguaje utilizado en las aulas no es inclusivo. & 1 & 2 & 3 & 4 & 5 \\
\hline 18 & La mayor parte de los problemas que ocurren en los patios son asuntos sin importancia & 1 & 2 & 3 & 4 & 5 \\
\hline 19 & Presentan dificultades y problemas similares las alumnas y los alumnos. & 1 & 2 & 3 & 4 & 5 \\
\hline 20 & Las expectativas que tenemos del alumnado no dependen del género. & 1 & 2 & 3 & 4 & 5 \\
\hline 21 & El liderazgo en mis clases suele ser asumido por algún chico. & 1 & 2 & 3 & 4 & 5 \\
\hline 22 & Las chicas recogen mejor y más cuidadosamente el material del aula que los chicos. & 1 & 2 & 3 & 4 & 5 \\
\hline 23 & $\begin{array}{l}\text { Cuando necesito que un estudiante me ayude a mover un material pesado prefiero } \\
\text { elegir a un chico. }\end{array}$ & 1 & 2 & 3 & 4 & 5 \\
\hline 24 & En ocasiones me siento incómodo al utilizar el masculino para referirme a las alumnas. & 1 & 2 & 3 & 4 & 5 \\
\hline 25 & Los chicos generan más problemas en los patios. & 1 & 2 & 3 & 4 & 5 \\
\hline 26 & Los problemas y dificultades ocasionados por los chicos son más fáciles de resolver. & 1 & 2 & 3 & 4 & 5 \\
\hline 27 & Es más probable que un gesto obsceno sea realizado por un chico. & 1 & 2 & 3 & 4 & 5 \\
\hline 28 & $\begin{array}{l}\text { Los iconos mediáticos que utilizo como ejemplo en las clases son por lo general } \\
\text { representativos de los hombres }\end{array}$ & 1 & 2 & 3 & 4 & 5 \\
\hline 29 & Las mujeres están mejor capacitadas para la docencia que los hombres. & 1 & 2 & 3 & 4 & 5 \\
\hline
\end{tabular}




\begin{tabular}{|c|c|c|c|c|c|c|}
\hline 30 & Trabajo poco con mis estudiantes la realidad transgénero y la homosexualidad. & 1 & 2 & 3 & 4 & 5 \\
\hline 31 & $\begin{array}{l}\text { Es más importante valorar el contenido que la forma en las tareas que me entregan } \\
\text { mis estudiantes. }\end{array}$ & 1 & 2 & 3 & 4 & 5 \\
\hline 32 & $\begin{array}{c}\text { La brecha digital (acceso y dominio de las TIC) entre hombres y mujeres es hoy en día } \\
\text { inexistente. }\end{array}$ & 1 & 2 & 3 & 4 & 5 \\
\hline 33 & Cuando reparto material a mis alumnas y alumnos lo hago de forma aleatoria & 1 & 2 & 3 & 4 & 5 \\
\hline 34 & $\begin{array}{c}\text { La motivación de la clase depende de los contenidos y la metodología que } \\
\text { seleccione. }\end{array}$ & 1 & 2 & 3 & 4 & 5 \\
\hline 35 & $\begin{array}{l}\text { Los enfoques competitivos en el aula consolidan la identidad de género. (unen a las } \\
\text { chicas entre sí y a los chicos entre sí) }\end{array}$ & 1 & 2 & 3 & 4 & 5 \\
\hline 36 & Los chicos son más chapuceros que las chicas al hacer las tareas. & 1 & 2 & 3 & 4 & 5 \\
\hline 37 & El coordinador TIC en los centros suele ser & 1 & 2 & 3 & 4 & 5 \\
\hline 38 & En mis clases los chicos suelen querer recibir primero el material. & 1 & 2 & 3 & 4 & 5 \\
\hline 39 & Los chicos siempre están más motivados en mis clases. & 1 & 2 & 3 & 4 & 5 \\
\hline 40 & $\begin{array}{c}\text { Generar un ambiente en clase en el que se fomente la participación de forma } \\
\text { competitiva beneficia más a los chicos }\end{array}$ & 1 & 2 & 3 & 4 & 5 \\
\hline \multicolumn{7}{|c|}{$\begin{array}{l}\text { Nota. DB: Ítems 1 1, 25, 26, 27. PM: Ítems 35, 38, } 39 \text { y 40. LIG: Ítems 1, 9,17, 21, 37. SGE: Ítems 6, 7, 15. PF: Ítems 22, 23, } 36 . \\
\text { *Todos los ítems perfilan en positivo. } \\
\text { **Los ítems en negrita forman parte de la escala final con } 19 \text { reactivos. Los ítems en cursiva fueron descartados después del AFE. }\end{array}$} \\
\hline
\end{tabular}

